\title{
Iron Uptake and Loading into Rice Grains
}

\author{
Khurram Bashir • Yasuhiro Ishimaru • \\ Naoko K. Nishizawa
}

Received: 20 April 2010 /Accepted: 1 June 2010 /Published online: 22 June 2010

(C) The Author(s) 2010. This article is published with open access at Springerlink.com

\begin{abstract}
Iron (Fe) is an important micronutrient for living organisms. Fe deficiency severely impairs plant growth and is a widespread human dietary problem, with particularly high numbers of affected children and females. Rice (Oryza sativa) is a source of energy for more than half of the world's population. Thus, understanding the mechanisms of Fe uptake and translocation in rice is of utmost importance in the development of rice varieties that are tolerant to low $\mathrm{Fe}$ availability and with high seed levels of Fe. In recent years, the mechanisms underlying Fe transport and homeostasis have been revealed, providing opportunities to increase the $\mathrm{Fe}$ content of rice grain. As excess $\mathrm{Fe}$ is toxic to cells, plants have developed sophisticated mechanisms to control Fe flow, making it difficult to alter $\mathrm{Fe}$ transport. Thus, choosing appropriate chelators and $\mathrm{Fe}$ transporters driven by appropriate promoters seems to be the key in developing rice that is tolerant to low Fe availability and which accumulates high grain levels of Fe. Many recent studies have been aimed at increasing the Fe content of rice. Here, we summarize these efforts and review recent progress in understanding the mechanisms of $\mathrm{Fe}$ transport.
\end{abstract}

Keywords Biofortification · Deoxymugineic acid · Iron . Nicotianamine $\cdot$ Rice $\cdot$ YSLs

K. Bashir · Y. Ishimaru $\cdot$ N. K. Nishizawa $(\bowtie)$

Graduate School of Agricultural and Life Sciences,

The University of Tokyo,

1-1-1 Yayoi, Bunkyo-Ku,

Tokyo 113-8657, Japan

e-mail: annaoko@mail.ecc.u-tokyo.ac.jp

\section{Introduction}

Iron $(\mathrm{Fe})$ is an essential micronutrient for all higher organisms. Fe deficiency is one of the most prevalent micronutrient deficiencies in humans, causing 0.8 million deaths annually (WHO 2002) and affecting approximately 2 billion people (Stoltzfus and Dreyfuss 1998). A common approach to mitigating Fe deficiency is to promote healthy food, supplementation, and food fortification (Haas et al. 2005), but poor families cannot afford these strategies, especially those living in developing countries. Moreover, $\mathrm{Fe}$ biofortification is difficult as many beneficial $\mathrm{Fe}$ compounds (e.g., $\mathrm{FeSO}_{4}$ ) are unpalatable, and less soluble Fe compounds are poorly absorbed (Hurrell 2002). Thus, increasing the $\mathrm{Fe}$ content of grain has great potential in combating $\mathrm{Fe}$ deficiency and will have a dramatic impact on human health (Clemens et al. 2002; Guerinot 2001). Increasing the $\mathrm{Fe}$ content of rice is a difficult task for several reasons. Although soil is abundant in $\mathrm{Fe}$, plants cannot utilize it as it is chiefly present as largely insoluble $\mathrm{Fe}(\mathrm{III})$ compounds. The fact that calcareous soils with a high $\mathrm{pH}$ account for $30 \%$ of the world's cultivated soils (Chen and Barak 1982) makes the situation worse. On the other hand, $\mathrm{Fe}$ is a transition metal that readily accepts and donates electrons. This property of Fe makes it essential for plants while, at the same time, making it toxic through the production of reactive oxygen species (Halliwell and Gutteridge 1986). Thus, plants have developed sophisticated mechanisms to absorb Fe from soil and to transport it from root to shoot and grain. To acquire Fe from soil, graminaceous plants secrete small molecules called mugineic acid family phytosiderophores (MAs) that solubilize Fe (Takagi 1976), and Fe (III)-MA complexes are readily taken up by specific transporter at the root surface. 
In recent years, much progress has been made in elucidating the MA biosynthetic pathway, identifying proteins that transport $\mathrm{Fe}$ in various forms, and uncovering the transcription factors controlling the Fe homeostasis. In this article, we summarize recent advances in our understanding of the molecular mechanisms underlying Fe uptake and transport in plants, efforts to increase plant tolerance to Fe deficiency, and attempts to increase the rice grain $\mathrm{Fe}$ content.

\section{Genes involved in Fe homeostasis}

The Fe homeostasis system in graminaceous plants utilizes a complex network of enzymes as well as Fe chelators like citrate, nicotianamine (NA), and deoxymugineic acid (DMA); transporters; and transcription factors. This network has been elucidated in great detail, although many parts of the puzzle have yet to be characterized. The genes controlling Fe homeostasis can be grouped into three broad categories: (1) enzymes, especially those involved in MA biosynthesis; (2) transporters of $\mathrm{Fe}$ in different forms; and (3) transcription factors controlling the expression of genes involved in $\mathrm{Fe}$ homeostasis.

\section{NA and DMA biosynthesis}

As mentioned above, graminaceous plants solubilize soil $\mathrm{Fe}$ by secreting $\mathrm{Fe}(\mathrm{III})$ chelators called MAs from their roots (Takagi 1976; Takagi et al. 1984). The resulting Fe(III)MA complexes are then absorbed into the roots by Fe(III)MA transporter.

The MA biosynthetic pathway has been characterized in detail. MAs are synthesized from L-Met (Mori and Nishizawa 1987). NA synthase (NAS) catalyzes the trimerization of $S$-adenosyl Met (SAM) to NA (Higuchi et al. 1994, 1999), which is then converted to a $3^{\prime}$-keto intermediate through the transfer of an amino group using NA aminotransferase (NAAT) (Kanazawa et al. 1995). The subsequent reduction of the 3'-carbon in the keto intermediate produces DMA, which is the first MA synthesized in this pathway. The biosynthetic pathway of all MAs is the same from L-Met to DMA; however, the subsequent steps differ depending on the plant species and cultivar (Ma et al. 1999). The production and secretion of MAs significantly increase in response to $\mathrm{Fe}$ deficiency, and tolerance to $\mathrm{Fe}$ deficiency in graminaceous plants is strongly correlated with the MAs secreted. For example, rice secretes only DMA in relatively low amounts and is thus sensitive to low $\mathrm{Fe}$ availability. In contrast, barley secretes large amounts of many MAs, including MA, 3-hydroxymugineic acid, and 3-epi-hydroxymugineic acid, and is therefore more tolerant to low Fe availability (Römheld and Marschner 1990; Singh et al. 1993).

The genes involved in MA biosynthesis have been characterized in rice; three genes, OsNAS1-3, are responsible for the trimerization of SAM (Inoue et al. 2003). OsNAAT1 converts NA to a $3^{\prime}$-keto intermediate in rice (Inoue et al. 2008), which is further converted to DMA by OsDMAS1 (Fig. 1; Bashir and Nishizawa 2006; Bashir et al. 2006). In barley, MAs are secreted in a diurnal fashion (Takagi et al. 1984). The diurnal secretion of MAs has not been fully characterized in rice, although the expression of MA family genes is believed to change in a diurnal fashion (Nozoye et al. 2004; Inoue et al. 2009).

In addition to these enzymes, the activity of rice glutathione reductase is also reported to be regulated by Fe deficiency (Bashir et al. 2007). Furthermore, the expression of genes with unknown functions is also regulated by $\mathrm{Fe}$ deficiency, and a knockout mutant for a mitochondrial Fe-regulated gene shows sensitivity to $\mathrm{Fe}$ deficiency compared to wild-type (WT) plants (Ishimaru et al. 2009).

\section{Transporters involved in Fe homeostasis}

Fe transport is a complex process involving a number of genes belonging to different families. Among the transporters involved in Fe homeostasis, the YSL family (Koike

Fig. 1 Biosynthetic pathway of MAs in rice. Three molecules of $S$-adenosyl methionine are combined by OsNAS1-3 to form NA. The amino group of NA is transferred by OsNAAT1, and the resultant $3^{\prime}$-keto intermediate is reduced to DMA by OsDMAS1. The subsequent steps differ with the plant species and cultivar.

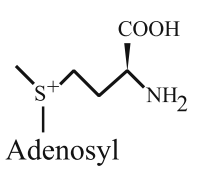

S-Adenosyl Methionine

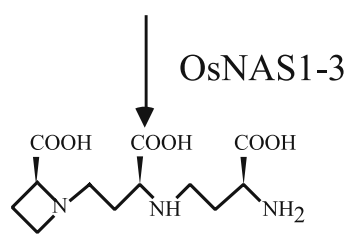

Nicotinanamine

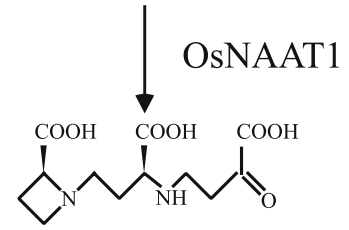

3"-Keto intermediate OsDMAS1

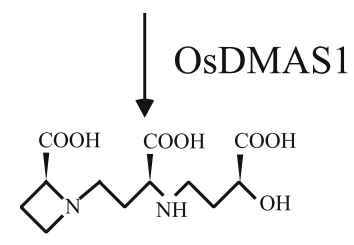

2`-Deoxymugineic Acid 
et al. 2004), rice Fe-regulated transporters (OsIRT1-2; Bughio et al. 2002; Ishimaru et al. 2006), and citrate transporters (Inoue et al. 2004; Yokosho et al. 2009) have been characterized in detail.

Maize yellow stripe 1 (YS1), which encodes an Fe(III)MA transporter (Curie et al. 2001), belongs to an oligopeptide family of transporters that function as protoncoupled symporters for various DMA-bound metals (Yen et al. 2001; Schaaf et al. 2004). The rice genome contains 18 putative YSL family genes (Koike et al. 2004), among which OsYSL2, OsYSL15, and OSYSL18 have been characterized in detail (Aoyama et al. 2009; Inoue et al. 2009; Ishimaru et al. 2010; Koike et al. 2004). The protein encoded by OSYSL15 transports Fe(III)-DMA from the rhizosphere to roots (Inoue et al. 2009), whereas OSYSL18 encodes a functional Fe(III)-DMA transporter involved in DMA-mediated $\mathrm{Fe}$ distribution in reproductive organs (Aoyama et al. 2009). In comparison, the translation product of OSYSL2 has been identified as an Fe-NA or Mn-NA transporter (Koike et al. 2004).

Rice also possesses the ability to transport $\mathrm{Fe}^{2+}$. In rice, two genes, OSIRT1 and OSIRT2, have been cloned and characterized. The proteins encoded by each gene localize to the plasma membrane and have been shown to complement the growth defect of a yeast Fe uptake mutant, confirming that they are functional Fe transporters (Bughio et al. 2002; Ishimaru et al. 2006). OsFRDL1, a member of the multidrug and toxic compound extrusion transporter family, is a citrate transporter localized to pericycle cells that is important for efficient $\mathrm{Fe}$ translocation to shoots (Inoue et al. 2004; Yokosho et al. 2009).

Transcription factors controlling the Fe homeostasis in rice

Transcription factors controlling the expression of genes involved in Fe uptake and translocation have also been identified. Two novel Fe deficiency-responsive cis-acting elements in barley, Fe deficiency-responsive elements 1 and 2 (IDE1 and IDE2; Kobayashi et al. 2003), were the first elements found to control the expression of genes involved in micronutrient homeostasis. When expressed in rice, IDE1 and IDE2 induce Fe deficiency-responsive gene expression in roots and leaves (Kobayashi et al. 2004). Sequences similar to IDE1 or IDE2 are present in various $\mathrm{Fe}$ deficiency-inducible promoters in barley, rice, tobacco, and Arabidopsis (Ducos et al. 2005; Kobayashi et al. 2003, 2005), suggesting that gene regulatory mechanisms involving IDEs are also functional in nongraminaceous plant species. The transcription factors for IDE1 and IDE2 have been characterized; IDE-binding factor 1 (IDEF1) and IDEF2 specifically bind to $I D E 1$ and $I D E 2$, respectively (Kobayashi et al. 2007; Ogo et al. 2008). IDEF1 and IDEF2 belong to uncharacterized branches of the plant-specific transcription factor families $\mathrm{ABI} 3 / \mathrm{VP} 1$ and $\mathrm{NAC}$, respectively. IDEF1 recognizes the sequence CATGC within $I D E 1$, whereas IDEF2 predominantly recognizes $\mathrm{CA}[\mathrm{A} / \mathrm{C}] \mathrm{G}[\mathrm{T} / \mathrm{C}][\mathrm{T} / \mathrm{C} / \mathrm{A}]$ $[\mathrm{T} / \mathrm{C} / \mathrm{A}]$ within $I D E 2$ as its core binding site. IDEF1 and $I D E F 2$ transcripts are constitutively expressed in rice roots and leaves (Kobayashi et al. 2010).

Moreover, the bHLH transcription factor gene OSIRO2, which binds specifically to CACGTGG and is regulated by $\mathrm{Fe}$ deficiency, has also been characterized (Ogo et al. 2006). OsIRO2 expression controls the expression of various $\mathrm{Fe}$ deficiency-induced genes in rice roots, including genes involved in MA biosynthesis (OsNAS1, OsNAS2, OsNAAT1, and OsDMAS1) and Fe(III)-MA transport. $\mathrm{OSIRO2}$ also affects the expression of $\mathrm{Fe}$ deficiencyinducible transcription factor genes possessing OsIRO2binding core sequences in their promoter regions (Ogo et al. 2007). OsIRO2 itself harbors multiple IDEFI-binding core sequences in its promoter region and is regulated by $I D E F 1$ (Kobayashi et al. 2007). IDEF1 expression is correlated with that of OSIRO2, OsYSL15, OsIRT1, OSYSL2, OSNAS1, $O S N A S 2, O s N A S 3$, and OSDMAS1 just after the onset of Fe deficiency, suggesting that $I D E F 1$ is essential for the early onset of the Fe deficiency response (Kobayashi et al. 2009).

\section{Fe homeostasis during germination}

Seed germination is a complex process that is regulated by various internal and external factors, including an intricate network of hormone signaling pathways, light, and water. Transporters that are involved in both vacuolar Fe influx and efflux have been identified, and they have been shown to be essential for germination and seedling development in Arabidopsis (Lanquar et al. 2005; Kim et al. 2006). Rice seeds contain not only Fe but also NA and DMA, and the amount of DMA is significantly higher than that of NA (Usuda et al. 2009; Masuda et al. 2009). During germination, Fe is mobilized along with NA or DMA, as revealed by an analysis of rice seeds grown without Fe (Takahashi et al. 2009).

Recent technological advances have made it possible to visualize Fe localization in seeds. The localization of Fe in germinating rice grains was observed using X-ray fluorescence imaging (Takahashi et al. 2009). During germination, $\mathrm{Fe}$ is detectable in the dorsal vascular bundle, aleurone layer, and endosperm (Fig. 2a), while in the embryo, it localizes to the scutellum and vascular bundle of the scutellum $12 \mathrm{~h}$ after sowing. Twenty-four hours after sowing, $\mathrm{Fe}$ remains detectable in the dorsal vascular bundle, is dispersed in the scutellum, and accumulates in the coleoptiles. Fe can also be seen in the epithelium and endosperm near the scutellum. Thirty-six hours after sowing, Fe can be observed in root tips. At this stage in 


\section{a}

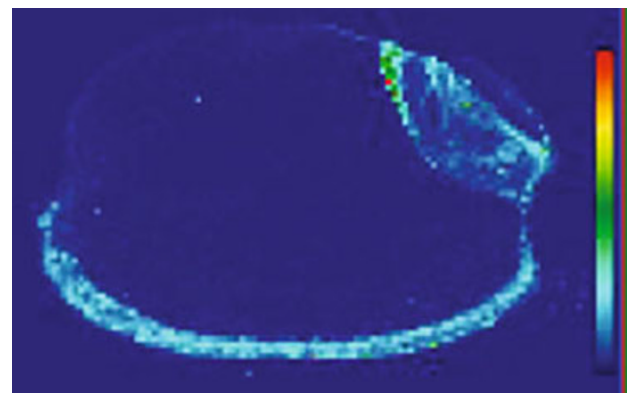

High

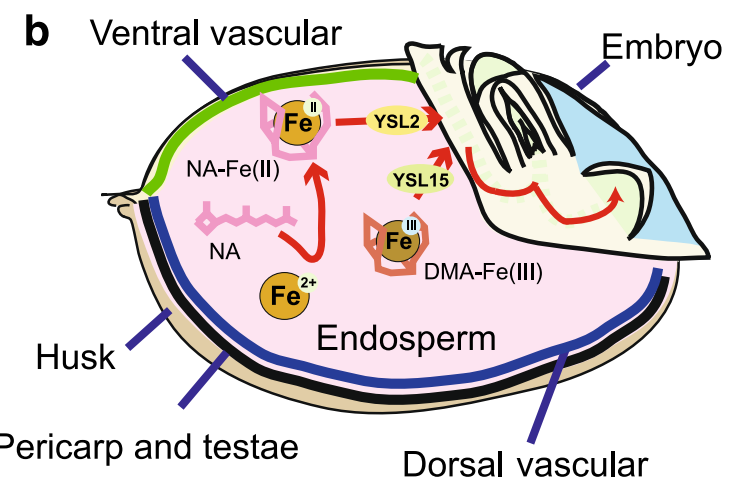

NA production area

DMA production area

NA and DMA production area

Fig. 2 Fe distribution during germination. Fe localization in seeds (a) and a schematic diagram (b) showing the production of NA and DMA and $\mathrm{Fe}$ distribution during germination. Modified from Nozoye et al. (2007).

the rice embryo, Fe localizes not only to the epithelium, scutellum, and coleoptile, but also to the leaf primordium and radicle (Takahashi et al. 2009).

More than $50 \%$ of Fe deficiency-inducible genes are highly expressed during germination, and $76 \%$ of $\mathrm{Fe}$ deficiency-inducible genes change their expression during seed germination. The expression of various Fe deficiencyrelated genes has been analyzed during germination through promoter-GUS analysis. IDEF1 and IDEF2 are expressed constitutively during germination in the embryo and endosperm (Kobayashi et al. 2010). Moreover, the expression of various genes involved in $\mathrm{Fe}$ homeostasis, including OSNAS2-3 and OSNAAT1, increases sharply after germination, indicating that NA and DMA production increases (Nozoye et al. 2007). The expression of the Fe transporters OsYSL2 (Fe-NA transporter) and OSIRT1 has been observed during germination. NA seems to be produced in the endosperm, while DMA is produced in the embryo, especially in the coleorhizae and bud scales. NA and DMA production is also overlapped in the embryo (Nozoye et al. 2007). Hence, NA and DMA are synthesized during germination and are involved in $\mathrm{Fe}$ translocation during germination. Furthermore, the expression of OsIRTI indicates that rice can utilize both $\mathrm{Fe}^{2+}$ and $\mathrm{Fe}^{3+}$ during germination (Nozoye et al. 2007; Takahashi et al. 2009). The role of $\mathrm{Fe}$ transporters during germination has also been studied using knockdown plants. In OSYSL2 RNAi plants, OsYSL2 does not translocate enough Fe to the roots and shoots of seedlings during germination, resulting in growth defects (Ishimaru et al. 2010). These data are supported by the finding that OSYSL2 is expressed mainly in the epithelium, vascular bundle of the scutellum, and leaf primordium (Nozoye et al. 2007), suggesting that OsYSL2 is also important for $\mathrm{Fe}$ translocation from seeds. The growth of OSYSL15 knockdown seedlings is arrested at early growth stages, including germination, and can be rescued by the supply of high levels of $\mathrm{Fe}$, indicating that OsYSL15 plays a crucial role in Fe uptake and homeostasis during early growth (Inoue et al. 2009). Changes in $\mathrm{Fe}$ localization, in the expression of Fe transporters, and growth defects in knockdown mutants of OsYSL2 and OsYSL15 suggest that these genes play a critical role in $\mathrm{Fe}$ translocation during germination (Fig. 2b).

\section{Fe uptake from soil}

As discussed above, soil contains abundant Fe; however, most of it is unavailable due to poor solubility. Therefore, plants have developed sophisticated and tightly regulated mechanisms for acquiring Fe from soil, and these can be grouped into two strategies (Marschner et al. 1986). Nongraminaceous plants lower the soil $\mathrm{pH}$ by enhancing phenolic and proton excretion into the rhizosphere, reducing the Fe to a more soluble ferrous form at the root surface by inducing the expression of ferric-chelate reductase and by transport of the resulting ferrous ions across the root plasma membrane through IRT1 (Vert et al. 2002).

The genes of the MA pathway are significantly regulated by Fe deficiency. OsNAS1-2 are tightly regulated in response to Fe deficiency in roots and shoots, while the expression of $O S N A S 3$, which is mainly expressed in Fe-sufficient shoots, is induced in roots but suppressed in shoots in response to $\mathrm{Fe}$ deficiency (Inoue et al. 2003). The expression of OsNAATI is strong in Fe-deficient roots, particularly in companion and pericycle cells (Inoue et al. 2008). The expression of OsDMAS1 is also responsive to Fe deficiency (Bashir et al. 2006). The expression patterns and localization of OSNAS12, OSNAAT1, and OSDMAS1 (root companion cells, pericycle, cortex, and epidermis/exodermis [Fig. 3a]) are quite similar, indicating that these genes work together to produce DMA for $\mathrm{Fe}$ acquisition and translocation.

Among $\mathrm{Fe}$ transporters, OsYSL15 is predominantly expressed in the epidermis/exodermis and phloem cells under conditions of Fe deficiency and only in phloem under Fe sufficiency (Inoue et al. 2009). The growth of OsYSL15 


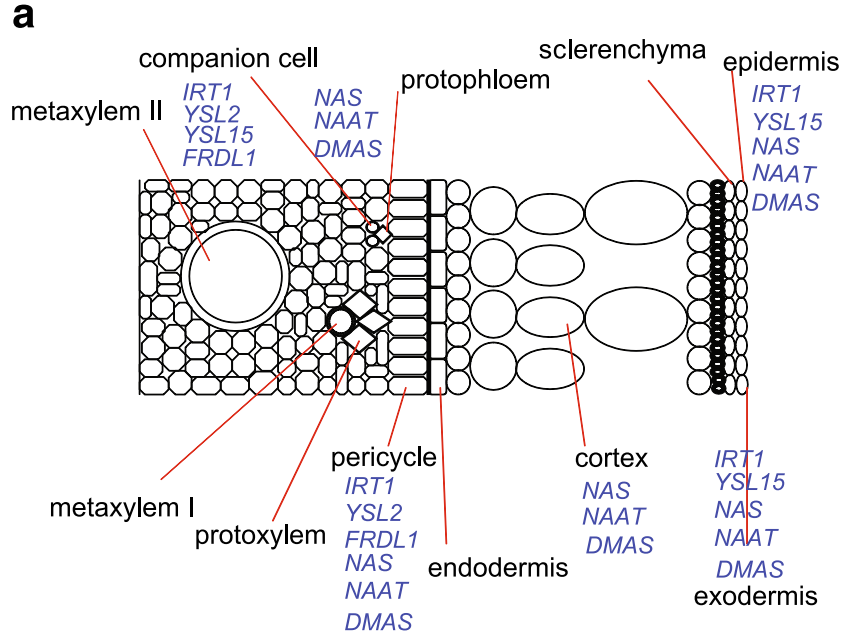

b

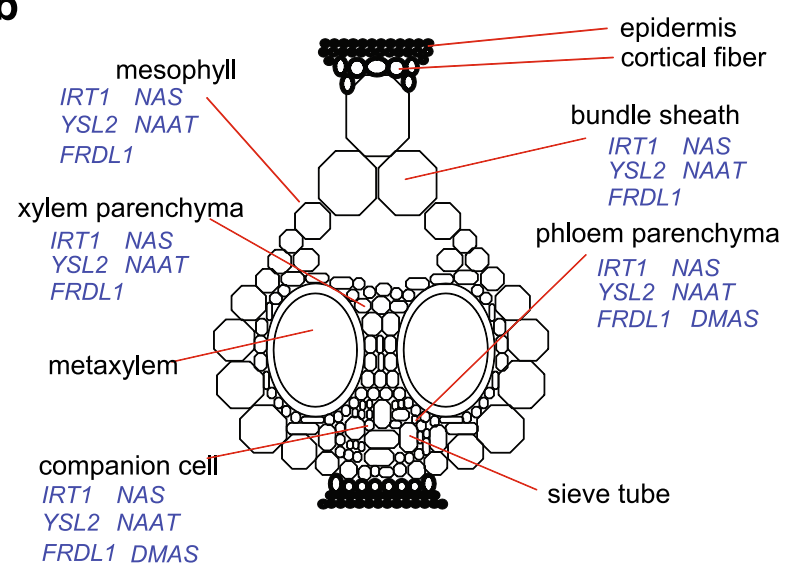

Fig. 3 Tissue-specific expression of Fe homeostasis-related genes in rice roots (a) and shoots (b).

RNAi seedlings is arrested during early growth and can be rescued by the supply of high levels of Fe (Inoue et al. 2009). Osysl15 T-DNA mutants are chlorotic under Fedeficient conditions and have reduced $\mathrm{Fe}$ concentrations in their shoots, roots, and seeds (Lee et al. 2009a), indicating that OsYSL15 plays a crucial role in $\mathrm{Fe}$ uptake and homeostasis (Inoue et al. 2009; Lee et al. 2009a).

Rice is unique in the sense that it utilizes both strategies. Besides secreting DMA, it also absorbs $\mathrm{Fe}^{2+}$, which is more abundant than $\mathrm{Fe}^{3+}$ under the submerged conditions to which rice is well adapted (Ishimaru et al. 2006). Although two homologs of ferric-chelate reductase are present in rice, the expression of these genes is not observed under Fedeficient or Fe-sufficient conditions, and the level of $\mathrm{Fe}^{3+}$ chelate reductase activity is very low compared to that in other plants (Ishimaru et al. 2006). As rice plants are adapted to grow under submerged conditions where $\mathrm{Fe}^{2+}$ is abundant, they may have lost the ability to reduce $\mathrm{Fe}^{3+}$ through the development of a functional $\mathrm{Fe}^{2+}$-regulated transporter, but not ferric-chelate reductase (Chang 2003; Ishimaru et al. 2006). OsIRT1 and OsIRT2 expression is observed only in Fe-deficient roots (Ishimaru et al. 2006). The existence of strategies I and II in rice allows it to utilize both ferrous and ferric iron, depending on their availability and environmental conditions.

\section{Fe homeostasis during vegetative and reproductive growth}

Fe transport from root to shoot and grain is essential for normal plant growth. In rice, $\mathrm{Fe}$ can be transported in various forms through the xylem and phloem, including Fecitrate, DMA-Fe(III), and NA-Fe(II). The knockout of the rice citrate transporter osfrdll results in chlorotic plants with low levels of $\mathrm{Fe}$ in the leaves and $\mathrm{Fe}$ precipitation in the root stele. Such mutants also show a reduced concentration of citrate and ferric iron in the xylem sap compared to WT rice. OsFRDL1 expression was first identified in cells involved in long-distance transport as well as in reproductive organs, and, unlike other Fe transporters, OsFRDL1 expression is not regulated by Fe availability (Inoue et al. 2004). These results suggest a role for OsFRDL1 in Fe homeostasis through the xylem.

Although DMA is utilized for Fe acquisition from soil, its role in internal Fe homeostasis cannot be overlooked. In rice and barley, DMA has been detected in shoots under Fesufficient conditions, and the amount of DMA increases under Fe deficiency. The amount of DMA is higher in rice leaves compared to barley leaves under Fe-sufficient and Fe-deficient conditions (Higuchi et al. 2001a), although barley secretes larger amounts of MAs. OsDMAS1 promoter-GUS activity was not observed in Fe-sufficient rice shoots, although it is expressed in portions of roots involved in long-distance transport, indicating that the DMA detected in Fe-sufficient rice leaves is translocated from roots in a complex with Fe (Bashir et al. 2006; Mori et al. 1991). On the other hand, DMA is at least partially synthesized in Fe-deficient shoots. The DMA synthesized in shoots is thought to be involved in Fe homeostasis in shoots and flowers and does not participate in the acquisition of $\mathrm{Fe}$ from soil (Bashir et al. 2006). Similar to roots, the expression of OsNASS, OSNAAT1, and OsDMAS1 is significantly overlapped in shoots, indicating that these genes work in coordination to produce DMA (Fig. 3b).

Among the 18 putative YSL family genes in rice, OsYSL 5-7, -14, and -17 are expressed in the epidermis, cortex, and stele of Fe-sufficient and Fe-deficient roots. On the other hand, the expression of OSYSL1-4, 9-11, and -18 was not observed in roots, irrespective of their $\mathrm{Fe}$ status. OSYSL12 is expressed in the cortex and stele under Fesufficient and Fe-deficient conditions, whereas OsYSL16 is expressed in the epi-/endodermis and cortex under Fe-sufficient condition and in the epi-/endodermis, cortex, 
and stele under Fe-deficient condition (Inoue et al. 2009). Although the expression of these genes is not significantly regulated by $\mathrm{Fe}$, at least in root tissues, the involvement of these genes in Fe homeostasis cannot be ruled out. YSL family genes also exist in nongraminaceous plants, where they transport metal-NA complexes (DiDonato et al. 2004). Phylogenically, the YSL family in plants can be grouped into four subgroups, and among these, OsYSL1, 3-4, 7-8, and 17-18 form a rice-specific group (Aoyama et al. 2009). In this group, only YSL18 has been characterized as an $\mathrm{Fe}-$ DMA transporter, and the rice-specific nature of this subgroup indicates that other genes may also transport DMA-metal complexes.

Among these genes, OsYSL2, OsYSL15, and OSYSL18 have been characterized in detail (Aoyama et al. 2009; Inoue et al. 2009; Ishimaru et al. 2010; Koike et al. 2004). As mentioned above, OsYSL15 transports Fe(III)-DMA from the rhizosphere to the roots and is involved in internal Fe homeostasis. OsYSL15 promoter-driven GUS expression was not only observed in leaf tissue but also at the flowering stage (Inoue et al. 2009). These results indicate that OSYSL15 is involved in $\mathrm{Fe}$ transport to rice grains. Furthermore, OsYSL18 encodes a functional Fe(III)-DMA transporter involved in DMA-mediated Fe distribution in reproductive organs, lamina joints, and phloem cells at the base of the leaf sheath (Aoyama et al. 2009).

NA, besides being an MA precursor, also serves as a chelator of divalent metals (Takahashi et al. 2003). In rice, OSYSL2 has been identified as an Fe-NA transporter (Koike et al. 2004). OsYSL2 is expressed at the reproductive stage, and it seems to contribute to Fe accumulation in seeds (Koike et al. 2004). Furthermore, OsYSL2 RNAi and 35S-OSYSL2 lines have been characterized (Ishimaru et al. 2010). At the vegetative stage, $\mathrm{Fe}$ and Mn concentrations decreased in the shoots of OsYSL2 RNAi plants, while the Fe concentration increased in the roots. At the reproductive stage, Fe translocation to the shoots and seeds was suppressed in OsYSL2 RNAi plants. The Fe and Mn concentrations also decreased in OSYSL2 RNAi seeds, especially in the endosperm. Surprisingly, the Fe concentration in OSYSL2 overexpressors was lower in seeds and shoots but higher in roots, compared with WT plants, indicating the role of OsYSL2 in Fe homeostasis.

\section{Transgenic approaches to mitigating Fe deficiency in plants and humans}

Rice tolerant to low Fe availability

Attempts to increase $\mathrm{Fe}$ uptake and ultimately increase tolerance to low $\mathrm{Fe}$ availability have utilized several approaches. Rice plants tolerant to low Fe availability were first produced through strengthening of the strategy II system with the heterologous expression of $H v N A A T$ genes. Plants harboring $H v N A A T A-B$ secrete large amounts of phytosiderophores compared to WT plants and are tolerant to alkaline soils (Takahashi et al. 2001). Furthermore, several genes from barley, including HvNAS1 and HvNAAT, in combination with $H v N A S I$ and IDS3 were introduced into rice to increase $\mathrm{Fe}$ deficiency tolerance under alkaline conditions. These plants showed significantly enhanced tolerance to low $\mathrm{Fe}$ availability when grown in calcareous soils (Suzuki et al. 2008). Furthermore, the yeast ferric reductase gene $F R E 1$ was reconstructed for expression in plants (refre1; reconstructed FRE1; Oki et al. 2004) and then mutagenized and screened, so that it could work under alkaline conditions, and the reconstructed gene was introduced into rice under control of the IRT1 promoter (Ishimaru et al. 2007). Transgenic rice harboring refrel/372 transported more Fe compared to vector controls, as revealed by positron emission tracer imaging system analysis. These transformants exhibited enhanced tolerance to low Fe availability in both hydroponic culture and calcareous soils and produced eight times more grain in calcareous soils compared to the vector control (Ishimaru et al. 2007). Rice plants overexpressing OsIRTI also showed enhanced tolerance to $\mathrm{Fe}$ deficiency in paddy fields. These plants accumulated higher levels of $\mathrm{Fe}$ and $\mathrm{Zn}$ in shoots, roots, and mature seeds, indicating that $O S I R T 1$ can be used to enhance micronutrient levels in rice grains (Lee and An 2009). The efforts to increase tolerance to $\mathrm{Fe}$ deficiency in alkaline soils have been reviewed recently (Kobayashi et al. 2008).

\section{Biofortification of rice with $\mathrm{Fe}$}

Different approaches have been adopted to increase the edible $\mathrm{Fe}$ in rice seed. In rice, as the pericarp, testae, aleurone layers, and embryos are removed during processing to improve the quality and shelf-life of the product, leaving only the endosperm as the edible part (Matsuo and Hoshikawa
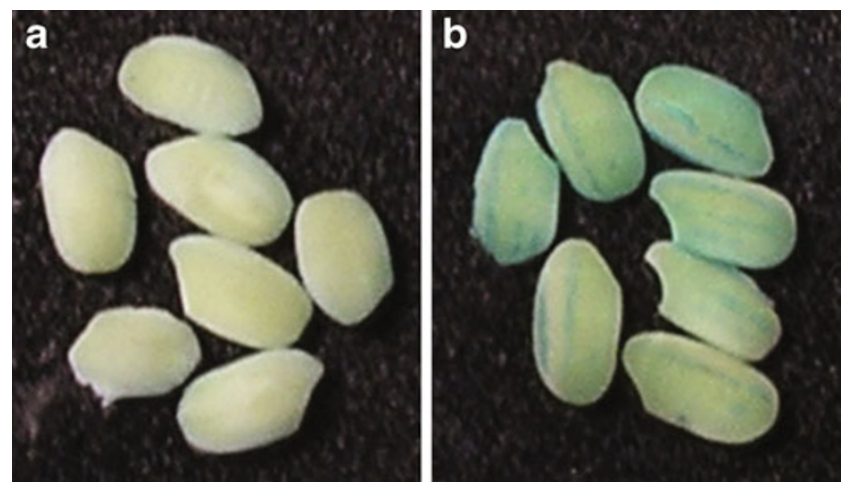

Fig. $4 \mathrm{Fe}$ localization in the seeds of WT plants as determined by Perl's staining. WT (a) and transgenic rice (b). 
1993), efforts have been focused on increasing the $\mathrm{Fe}$ content of the rice endosperm. The first attempt to increase the endosperm $\mathrm{Fe}$ content in rice employed the overexpression of ferritin (Goto et al. 1999), which has the unique ability to store up to 4,500 Fe atoms (Harrison and Arosio 1996). Plants expressing ferritin accumulated more $\mathrm{Fe}$ in the seed endosperm compared to WT plants (Goto et al. 1999; Vasconcelos et al. 2003). Transgenic rice plants expressing soybean ferritin under control of the seed-specific rice Gluterin-B1 promoter accumulated up to three times more seed Fe compared to WT plants (Goto et al. 1999), although $\mathrm{Fe}$ accumulation did not parallel the high expression level of ferritin in rice seeds (Qu et al. 2005). A twofold increase in the $\mathrm{Fe}$ content of rice seeds carrying FERRITIN from Phaseolus vulgaris has been reported (Lucca et al. 2001). However, an attempt to increase the bioavailability of Fe by expressing a thermo-tolerant phytase from Aspergillus fumigatus and a cysteine-rich metallothionine-like protein in rice met with limited success (Lucca et al. 2001). These results indicate that in addition to increased Fe storage in seeds, efforts to enhance $\mathrm{Fe}$ uptake from soil and its translocation are essential to further increase the $\mathrm{Fe}$ content of rice endosperm. Rice transformed with barley IDS3 (a mugineic acid-synthesizing dioxygenase gene) accumulated more $\mathrm{Fe}$ in the grain (Masuda et al. 2008). Transgenic lines harboring HvNAS1 driven by the $35 \mathrm{~S}$ or actin1 promoter accumulated more $\mathrm{Fe}$ and $\mathrm{Zn}$ in polished $\mathrm{T} 2$ seeds, showing a positive correlation between $\mathrm{Fe}$ and NA/DMA concentrations in seeds (Masuda et al. 2009). These results suggest that NA overproduction enhances the translocation of $\mathrm{Fe}$ and $\mathrm{Zn}$ to rice grains. Higuchi et al. (2001a, b) produced rice lines expressing $H v N A S 1$, resulting in an increased NA content in the roots and leaves under Fe-sufficient conditions. However, the seed $\mathrm{Fe}$ and $\mathrm{Zn}$ concentrations did not increase in plants grown under Fe-deficient conditions in calcareous paddy fields or under Fe-sufficient conditions in andosol paddy fields (Masuda et al. 2008; Suzuki et al. 2008). This difference in $\mathrm{Fe}$ accumulation in rice seeds is thought to be related to expression of the NAS transgene and subsequent NA accumulation. Under Fe-sufficient conditions, the NA concentration in 35S-HvNAS1 lines was higher than that in lines harboring the HvNAS1 genomic fragment (Higuchi et al. 2001a). Moreover, in 35S-HvNAS1 lines, the NAS transgene was presumably expressed throughout the rice plant, which would promote widespread $\mathrm{Fe}$ circulation and bioavailability.

Transgenic plants expressing AtNAS1 and Pvferritin simultaneously accumulated six times more $\mathrm{Fe}$ in the endosperm than WT plants, showing that this combination exerts a synergistic effect on Fe uptake and storage (Wirth et al. 2009). However, a similar approach using HvNAS1 in combination with soybean ferritin did not increase the seed Fe content (Masuda et al. 2009). Activation tagging for
OsNAS3 also resulted in increased $\mathrm{Fe}$ accumulation in roots, shoots, and seeds (Lee et al. 2009b). OsNAS3activated plants grown on a paddy field accumulated three times more Fe than WT plants and 9.6 times more NA in their seeds. This $\mathrm{Fe}$ is bioavailable, as shown by a feeding test using mice (Lee et al. 2009b).

In addition to increased $\mathrm{Fe}$ storage and uptake, increased $\mathrm{Fe}$ translocation may significantly enhance the seed $\mathrm{Fe}$ content, given that the strong expression of OSIRT1 in rice resulted in a marginal increase in seed $\mathrm{Fe}$ (Lee and An 2009). Rice plants overexpressing OsYSL15 accumulated up to $29 \%$ more Fe than WT plants (Lee et al. 2009a). Recently, it was demonstrated that the expression of OSYSL2, if driven by a suitable promoter, resulted in a significant increase in grain Fe (Ishimaru et al. 2010). The expression of OsYSL2, when controlled by the sucrose transporter promoter, increased the $\mathrm{Fe}$ concentration in polished rice up to 4.4-fold compared to WT, indicating that the control of gene expression in terms of temporal and spatial expression could lead to the creation of high Fe rice seeds (Fig. 4).

\section{Conclusions}

Despite significant effort and progress, the problem of $\mathrm{Fe}$ deficiency cannot be solved immediately, and there is still room for the development of better rice varieties through a combinatorial approach. For example, although lines overexpressing OsNAS1, OsNAAT1, refre1, OsIRO2, or IDEF2 show enhanced tolerance to low $\mathrm{Fe}$ availability, the combination of these genes may further enhance tolerance to $\mathrm{Fe}$ deficiency in alkaline soils. In particular, the expression of DMAS1 regulated by an appropriate promoter may significantly enhance tolerance to Fe deficiency by increasing MA production. Similarly, different approaches to achieve high Fe rice may be combined to breed varieties that accumulate more $\mathrm{Fe}$ in the rice endosperm.

Acknowledgments We greatly acknowledge the technical support by Dr. Tomoko Nozoye (The University of Tokyo). This work was supported by a grant from the Ministry of Agriculture, Forestry, and Fisheries of Japan (Green Technology Project IP-5003).

Open Access This article is distributed under the terms of the Creative Commons Attribution Noncommercial License which permits any noncommercial use, distribution, and reproduction in any medium, provided the original author(s) and source are credited.

\section{References}

Aoyama T, Kobayashi T, Takahashi M, Nagasaka S, Usuda K, Kakei $\mathrm{Y}$, et al. OsYSL18 is a rice iron(III)-deoxymugineic acid transporter specifically expressed in reproductive organs and phloem of lamina joints. Plant Mol Biol. 2009;70:681-92. 
Bashir K, Inoue H, Nagasaka S, Takahashi M, Nakanishi H, Mori S, et al. Cloning and characterization of deoxymugineic acid synthase genes from graminaceous plants. J Biol Chem. 2006;281:32395402.

Bashir K, Nishizawa NK. Deoxymugineic acid synthase; a gene important for $\mathrm{Fe}$-acquisition and homeostasis. Plant Signal Behav. 2006;1:290-2.

Bashir K, Nagasaka S, Itai RN, Kobayshi T, Takahashi M, Nakanishi $\mathrm{H}$, et al. Expression and enzyme activity of glutathione reductase is upregulated by Fe-deficiency in graminaceous plants. Plant Mol Biol. 2007;65:277-84.

Bughio N, Yamaguchi H, Nishizawa NK, Nakanishi H, Mori S. Cloning an iron-regulated metal transporter from rice. J Exp Bot. 2002;53:1677-82.

Chang TT. In: Smith CW, Dilday R-H, editors. Rice: origin, history, technology, and production. Hoboken, NJ: Wiley; 2003. p. 1-25.

Chen Y, Barak P. Iron nutrition of plants in calcareous soils. Adv Agron. 1982;35:217-40.

Clemens S, Palmgren MG, Kra"mer U. A long way ahead: understanding and engineering plant metal accumulation. Trends Plant Sci. 2002;7:309-15.

Curie C, Panaviene Z, Loulergue C, Dellaporta SL, Briat JF, Walker EL. Maize yellow stripe 1 encodes a membrane protein directly involved in Fe(III) uptake. Nature. 2001;409:346-9.

DiDonato Jr RJ, Roberts LA, Sanderson T, Eisley RB, Walker EL. Arabidopsis yellow stripe-like2 (YSL2): a metal-regulated gene encoding a plasma membrane transporter of nicotianamine-metal complexes. Plant J. 2004;39:403-14.

Ducos E, Fraysse ÅS, Boutry M. NtPDR3, an iron-deficiency inducible ABC transporter in Nicotiana tabacum. FEBS Lett. 2005;579:6791-5.

Goto F, Yoshihara T, Shigemoto N, Toki S, Takaiwa F. Iron fortification of rice seed by the soybean ferritin gene. Nat Biotechnol. 1999;17:282-6.

Guerinot ML. Improving rice yields-ironing out the details. Nat Biotechnol. 2001;19:417-8.

Haas JD, Beard JL, Murray-Kolb LE, del Mundo AM, Felix A, Gregorio GB. Iron-biofortified rice improves the iron stores of non-anemic Filipino women. J Nutr. 2005;135:2823-30.

Halliwell B, Gutteridge JM. Oxygen free radicals and iron in relation to biology and medicine: some problems and concepts. Arch Biochem Biophys. 1986;246:501-14.

Harrison PM, Arosio P. The ferritins: molecular properties, iron storage function and cellular regulation. Biochem Biophys Acta. 1996;1275:161-203.

Higuchi K, Kanazawa K, Nishizawa NK, Chino M, Mori S. Purification and characterization of nicotianamine synthase from Fe deficient barley roots. Plant Soil. 1994;165:173-9.

Higuchi K, Suzuki K, Nakanishi H, Yamaguchi H, Nishizawa NK, Mori S. Cloning of nicotianamine synthase genes, novel genes involved in the biosynthesis of phytosiderophores. Plant Physiol. 1999;119:471-9.

Higuchi K, Watanabe S, Takahashi M, Kawasaki S, Nakanishi H, Nishizawa NK, et al. Nicotianamine synthase gene expression differs in barley and rice under Fe-deficient conditions. Plant J. 2001a;25:159-67.

Higuchi K, Takahashi M, Nakanishi H, Kawasaki S, Nishizawa NK, Mori $\mathrm{S}$. Analysis of transgenic rice containing barley nicotianamine synthase gene. Soil Sci Plant Nutr. 2001b;47:315-22.

Hurrell RF. Fortification: overcoming technical and practical barriers. J Nutr. 2002;132:806-12.

Inoue $\mathrm{H}$, Higuchi $\mathrm{K}$, Takahashi $\mathrm{M}$, Nakanishi $\mathrm{H}$, Mori $\mathrm{S}$, Nishizawa NK. Three rice nicotianamine synthase genes, OsNAS1, OsNAS2, and $O S N A S 3$ are expressed in cells involved in long-distance transport of iron and differentially regulated by iron. Plant $\mathrm{J}$. 2003;36:366-81.
Inoue $\mathrm{H}$, Mizuno D, Takahashi M, Nakanishi H, Mori S, Nishizawa NK. A rice FRD3-like $(O s R F D L 1)$ gene is expressed in the cells involved in long-distance transport. Soil Sci Plant Nutr. 2004;50: $1133-40$.

Inoue $\mathrm{H}$, Takahashi M, Kobayashi T, Suzuki M, Nakanishi H, Mori S, et al. Identification and localisation of the rice nicotianamine aminotransferase gene OsNAAT1 expression suggests the site of phytosiderophore synthesis in rice. Plant Mol Biol. 2008;66:193203.

Inoue H, Kobayashi T, Nozoye T, Takahashi M, Kakei Y, Suzuki K, et al. Rice OsYSL15 is an iron-regulated iron(III)-deoxymugineic acid transporter expressed in the roots and is essential for iron uptake in early growth of the seedlings. J Biol Chem. 2009;284: 3470-9.

Ishimaru Y, Suzuki M, Tsukamoto T, Suzuki K, Nakazono M, Kobayashi $\mathrm{T}$, et al. Rice plants take up iron as an $\mathrm{Fe}^{3+}$. phytosiderophore and as $\mathrm{Fe}^{2+}$. Plant J. 2006;45:335-46.

Ishimaru Y, Kim S, Tsukamoto T, Oki H, Kobayashi T, Watanabe S, et al. Mutational reconstructed ferric chelate reductase confers enhanced tolerance in rice to iron deficiency in calcareous soil. Proc Natl Acad Sci USA. 2007;104:7373-8.

Ishimaru Y, Bashir K, Fujimoto M, An G, Nakanishi Itai R, Tsutsumi $\mathrm{N}$, et al. Rice-specific mitochondrial iron-regulated gene $(M I R)$ plays an important role in iron homeostasis. Mol Plant. 2009;2:1059-66.

Ishimaru Y, Masuda H, Bashir K, Inoue H, Tsukamoto T, Takahashi $\mathrm{M}$, et al. Rice metal-nicotianamine transporter, OsYSL2, is required for long distance transport of iron and manganese. Plant J. 2010;62:379-90.

Kanazawa K, Higuchi K, Nishizawa NK, Fushiya S, Mori S. Detection of two distinct isozymes of nicotianamine aminotransferase in $\mathrm{Fe}$ deficient barley roots. J Exp Bot. 1995;46:1241-4.

Kim SA, Punshon T, Lanzirotti A, Li L, Alonso JM, Ecker JR, et al. Localization of iron in arabidopsis seed requires the vacuolar membrane transporter VIT1. Science. 2006;314:1295-8.

Kobayashi T, Nakayama Y, Itai RN, Nakanishi H, Yoshihara T, Mori $\mathrm{S}$, et al. Identification of novel cis-acting elements, IDE1 and IDE2, of the barley IDS2 gene promoter conferring iron deficiency-inducible, root-specific expression in heterogeneous tobacco plants. Plant J. 2003;36:780-93.

Kobayashi T, Nakayama Y, Takahashi M, Inoue H, Nakanishi H, Yoshihara $\mathrm{T}$, et al. Construction of artificial promoters highly responsive to iron deficiency. Soil Sci Plant Nutr. 2004;50:116775 .

Kobayashi T, Suzuki M, Inoue H, Itai RN, Takahashi M, Nakanishi H, et al. Expression of iron-acquisition-related genes in irondeficient rice is co-ordinately induced by partially conserved iron-deficiency-responsive elements. J Exp Bot. 2005;56:130516

Kobayashi T, Ogo Y, Itai RN, Nakanishi H, Takahashi M, Mori S, et al. The novel transcription factor IDEF1 regulates the response to and tolerance of iron deficiency in plants. Proc Natl Acad Sci USA. 2007;104:19150-5.

Kobayashi T, Nakanishi H, Takahashi M, Mori S. Generation and field trials of transgenic rice tolerant to iron deficiency. Rice. 2008;1: 144-53.

Kobayashi T, Nakanishi Itai R, Ogo Y, Kakei Y, Nakanishi H, Takahashi $\mathrm{M}$, et al. The rice transcription factor IDEF1 is essential for the early response to iron deficiency and induces vegetative expression of late embryogenesis abundant genes. Plant J. 2009;60:948-61.

Kobayashi T, Ogo Y, May SA, Nozoye T, Itai RN, Nakanishi H, et al. The spatial expression and regulation of transcription factors IDEF1 and IDEF2. Ann Bot. 2010. doi:10.1093/aob/mcq002.

Koike S, Inoue H, Mizuno D, Takahashi M, Nakanishi H, Mori S, et al. OsYSL2 is a rice metal-nicotianamine transporter that is 
regulated by iron and expressed in the phloem. Plant J. 2004;39:415-24.

Lanquar V, Lelièvre F, Bolte S, Hames C, Alcon C, Neumann D, et al. Mobilization of vacuolar iron by AtNRAMP3 and AtNRAMP4 is essential for seed germination on low iron. EMBO. 2005;24:404151.

Lee S, An G. Over-expression of OsIRT1 leads to increased iron and zinc accumulations in rice. Plant Cell Environ. 2009;32:408-16.

Lee S, Chiecko JC, Kim SA, Walker EL, Lee Y, Guerinot ML, et al. Disruption of OsYSL15 leads to iron inefficiency in rice plants. Plant Physiol. 2009a; 150:786-800.

Lee S, Jeon US, Lee SJ, Kim YK, Persson DP, Husted S, et al. Iron fortification of rice seeds through activation of the nicotianamine synthase gene. Proc Natl Acad Sci USA. 2009b;106:22014-9.

Lucca P, Hurrell R, Potrykus I. Genetic engineering approaches to improve the bioavailability and the level of iron in rice grains. Theor Appl Genet. 2001;102:392-7.

Ma JF, Taketa S, Chang YC, Takeda K, Matsumoto H. Biosynthesis of phytosiderophores in several Triticeae species with different genomes. J Exp Bot. 1999;50:723-6.

Marschner H, Römheld V, Kissel M. Different strategies in higher plants in mobilization and uptake of iron. J Plant Nutr. 1986;9:695713.

Masuda H, Suzuki M, Morikawa KC, Kobayashi T, Nakanishi H, Takahashi $\mathrm{M}$, et al. Increase in iron and zinc concentrations in rice grains via the introduction of barley genes involved in phytosiderophore synthesis. Rice. 2008;1:100-8.

Masuda H, Usuda K, Kobayashi T, Ishimaru Y, Kakei Y, Takahashi $\mathrm{M}$, et al. Overexpression of the barley nicotianamine synthase gene HvNAS1 increases iron and zinc concentrations in rice grains. Rice. 2009;2:155-66.

Matsuo T, Hoshikawa K. Science of the rice plant. Vol I. Tokyo: Food and Agriculture Policy Research Center; 1993. p. 381.

Mori S, Nishizawa N. Methionine as a dominant precursor of phytosiderophores in Graminaceae: plants. Plant Cell Physiol. 1987;28:1081-92.

Mori S, Nishizawa NK, Hayashi H, Chino M, Yoshimura E, Ishihara J. Why are young rice plants highly susceptible to Fe-deficiency? Plant Soil. 1991;130:143-56.

Nozoye T, Itai RN, Nagasaka S, Takahashi M, Nakanishi H, Mori S, et al. Diurnal changes in the expression of genes that participate in phytosiderophore synthesis in rice. Soil Sci Plant Nutr. 2004;50: $1125-31$.

Nozoye T, Inoue H, Takahashi M, Ishimaru Y, Nakanishi H, Mori S, et al. The expression of iron homeostasis-related genes during rice germination. Plant Mol Biol. 2007;64:35-47.

Ogo Y, Itai RN, Nakanishi H, Inoue H, Kobayashi T, Suzuki M, et al. Isolation and characterization of IRO2, a novel iron-regulated bHLH transcription factor in graminaceous plants. J Exp Bot. 2006;57:2867-78

Ogo Y, Itai RN, Nakanishi H, Kobayashi T, Takahashi M, Mori S, et al. The rice bHLH protein OsIRO2 is an essential regulator of the genes involved in Fe uptake under Fe-deficient conditions. Plant J. 2007;51:366-77.

Ogo Y, Kobayashi T, Itai RN, Nakanishi H, Kakei Y, Takahashi M, et al. A novel NAC transcription factor IDEF2 that recognizes the iron deficiency-responsive element 2 regulates the genes involved in iron homeostasis in plants. J Biol Chem. 2008;283: 13407-17.

Oki H, Kim S, Nakanishi H, Takahashi M, Yamaguchi H, Mori S, et al. Directed evolution of yeast ferric reductase to produce plants with tolerance to iron deficiency in alkaline soils. Soil Sci Plant Nutr. 2004;50:1159-65.

Qu LQ, Yoshihara T, Ooyama A, Goto F, Takaiwa F. Iron accumulation does not parallel the high expression level of ferritin in transgenic rice seeds. Planta. 2005;222:225-33.

Römheld V, Marschner H. Genotypical differences among graminaceous species in release of phytosiderophores and uptake of iron phytosiderophores. Plant Soil. 1990;123:147-53.

Schaaf G, Ludewig U, Erenoglu BE, Mori S, Kitahara T, von Wire'n N. ZmYS1 Functions as a proton-coupled symporter for phytosiderophore- and nicotianamine-chelated metals. J Biol Chem. 2004;279:9091-6.

Singh K, Chino M, Nishizawa NK, Ohata T, Mori S. Genetic aspects of plant mineral nutrition. In Randall RJ, Delhaize E, Richards RA, Munns R (eds) Kluwer Academic, pp. 335-9; 1993.

Stoltzfus RJ, Dreyfuss ML. Guidelines for the use of iron supplements to prevent and treat iron deficiency anemia. Washington (DC): ILSI Press; 1998.

Suzuki M, Morikawa KC, Nakanishi H, Takahashi M, Saigusa M, Mori $\mathrm{S}$, et al. Transgenic rice lines that include barley genes have increased tolerance to low iron availability in a calcareous paddy soil. Soil Sci Plant Nutr. 2008;54:77-85.

Takagi S. Naturally occurring iron-chelating compounds in oat and rice-root washings. Soil Sci Plant Nutr. 1976;22:423-33.

Takagi S, Nomoto K, Takemoto S. Physiological aspect of mugineic acid, a possible phytosiderophore of graminaceous plants. J Plant Nutr. 1984;7:469-77.

Takahashi M, Nakanishi H, Kawasaki S, Nishizawa NK, Mori S. Enhanced tolerance of rice to low iron availability in alkaline soils using barley nicotianamine aminotransferase genes. Nat Biotechnol. 2001;19:466-9.

Takahashi M, Terada Y, Nakai I, Nakanishi H, Yoshimura E, Mori S, et al Role of nicotianamine in the intracellular delivery of metals and plant reproductive development. Plant Cell. 2003;15:1263-80.

Takahashi M, Nozoye T, Kitajima B, Fukuda N, Hokura N, Terada Y, et al. In vivo analysis of metal distribution and expression of metal transporters in rice seed during germination process by microarray and X-ray fluorescence imaging of $\mathrm{Fe}, \mathrm{Zn}, \mathrm{Mn}$, and Cu. Plant Soil. 2009;325:39-51.

Usuda K, Wada Y, Ishimaru Y, Kobayashi T, Takahashi M, Nakanishi $\mathrm{H}$, et al. Genetically engineered rice containing larger amounts of nicotianamine to enhance the antihypertensive effect. Plant Biotech J. 2009;7:87-95.

Vasconcelos M, Datta K, Oliva N, Khalekuzzaman M, Torrizo L, Krishnan S, et al. Enhanced iron and zinc accumulation in transgenic rice with the ferritin gene. Plant Sci. 2003;164:371-8.

Vert G, Grotz N, Dédaldéchamp F, Gaymand F, Guerinot M, Briat J, et al. IRT1, an arabidopsis transporter essential for iron uptake from the soil and for plant growth. Plant Cell. 2002;14:1223-33.

Wirth J, Poletti S, Aeschlimann B, Yakandawala N, Drosse B, Osorio $\mathrm{S}$, et al. Rice endosperm iron biofortification by targeted and synergistic action of nicotianamine synthase and ferritin. Plant Biotech J. 2009;7:1-14.

World Health Organization (WHO). World health report reducing risks, promoting healthy life. Geneva: WHO; 2002.

Yen MR, Tseng YH, Saier Jr MH. Maize yellow stripe1, an ironphytosiderophore uptake transporter, is a member of the oligopeptide transporter (OPT) family. Microbiology. 2001;147:2881-3.

Yokosho K, Yamaji N, Ueno D, Mitani N, Ma JF. OsFRDL1 is a citrate transporter required for efficient translocation of iron in rice. Plant Physiol. 2009;149:297-305. 